\title{
Temperature dependence of electric resistance and magnetoresistance of pressed nanocomposites of multilayer nanotubes with the structure of nested cones
}

\author{
V.I. Tsebro ${ }^{\mathrm{a}, 1}$ O.E. Omel'yanovskii ${ }^{\mathrm{a}}$, E.F. Kukovitskii ${ }^{\mathrm{b}}$, \\ N.A. Sainov ${ }^{b}$, N.A. Kiselev ${ }^{c}$, D.N. Zakharov ${ }^{c}$, \\ ${ }^{a}$ P.N. Lebedev Physics Institute, Russian Academy of Sciences, 117924 Moscow, \\ Russia \\ ${ }^{\mathrm{b}}$ Kazan Physicotechnical Institute, 420029 Kazan, Russia \\ ${ }^{\mathrm{c}}$ A.V. Shubnikov Institute of Crystallography, Russian Academy of Sciences, \\ 177333 Moscow, Russia
}

\begin{abstract}
Bulk samples of carbon multilayer nanotubes with the structure of nested cones (fishbone structure) suitable for transport measurements, were prepared by compressing under high pressure $(\sim 25 \mathrm{kbar})$ a nanotube precursor synthesized through thermal decomposition of polyethylene catalyzed by nickel. The structure of the initial nanotube material was studied using high-resolution transmission electron microscopy. In the low-temperature range $(4.2-100 \mathrm{~K})$ the electric resistance of the samples changes according to the law $\ln \rho \propto\left(T_{0} / T\right)^{1 / 3}$, where $T_{0} \sim 7 \mathrm{~K}$. The measured magnetoresistance is quadratic in the magnetic field and linear in the reciprocal temperature. The measurements have been interpreted in terms of two-dimensional variable-range hopping conductivity. It is suggested that the space between the inside and outside walls of nanotubes acts as a two-dimensional conducting medium. Estimates suggest a high value of the density of electron states at the Fermi level of about $5 \times 10^{21} \mathrm{eV}^{-1} \mathrm{~cm}^{-3}$.
\end{abstract}

Investigations of electric transport properties of carbon nanotubes has attracted great attention recently. According to theoretical concepts [1], an isolated nanotube can be either a metal, or semimetal, or insulator, depending on such structural parameters as its diameter, chirality, and the number of concentric layers in it. Despite enormous difficulties in measurements of electric parameters of isolated nanotubes or nanotube bundles, several attempts

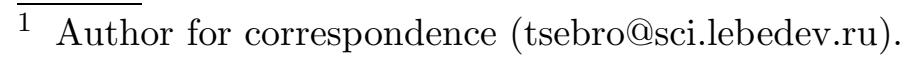


undertaken recently have been successful [2-4]. The latest published measurements [4] clearly indicate the presence of both metallic and insulating nanotubes in a single set of samples prepared in the same conditions. The authors emphasized that each multilayer nanotube manifested its specific conducting properties, thus indicating a strong correlation between structural and electric parameters.

In this connection, it is interesting to study, in addition to the transport properties of isolated carbon nanotubes, the conducting properties of bulk nanotube materials, in which contacts between nanotubes and/or their sections are randomly distributed. In our previous publication [5] we reported on the conductivity temperature dependence and structure (see also Ref. [6]) of carbon nanotube films fabricated by evaporating graphite in an electron beam. The data of those experiments were interpreted in terms of a three-dimensional model of hopping conductivity with a Coulomb gap about the Fermi level (the resistivity was described by the law $\left.\ln \rho \propto\left(T_{0} / T\right)^{1 / 2}\right)$. The density of states at the Fermi level for films that contained, as shown by structural investigations, mostly one-layer carbon nanotubes (isolated or assembled in bundles) and had a relatively high conductivity was estimated to be $g(\mu) \sim 10^{21} \mathrm{eV}^{-1} \mathrm{~cm}^{-3}$. On the other hand, films containing multilayer carbon nanotubes were characterized by fairly large values of resistivity, which changed with temperature to Mott's law, $\ln \rho \propto\left(T_{0} / T\right)^{1 / 4}$. In this case, estimates of the density of states, $g(\mu) \sim 10^{18} \mathrm{eV}^{-1} \mathrm{~cm}^{-3}$, corresponded to $g(\mu)$ for amorphous carbon. Amorphous carbon in significant quantities was detected on the outside surfaces of multilayer nanotubes in such films by electron microscopy [5,6], and it seems that the conductivity of such films can be attributed to the presence of carbon.

It is well known that, in addition to one-layer and multilayer nanotubes with walls made of coaxial carbon layers, there are nanotubes whose walls consist of nested truncated cones (these are the so-called fishbone-type structures [7]). Such nanocones are usually detected at the ends of carbon nanotubes, but can also exist in the form of independent objects among products of arc discharges in a helium atmosphere [8], commonly used in synthesizing carbon nanotubes.

In our recent work [9-11] we demonstrated that thermal decomposition of polyethylene with nickel used as a catalyst is a fairly efficient technique for fabrication of large quantities of fishbone nanotubes. This technique allows one to manufacture in a relatively short time considerable quantities (several grams) of fairly homogeneous nanotube material. According to the data of thermal analysis in oxiding atmosphere, the nickel content in this material is less then $15 \%$ by mass. Nickel is present in the material in the form of nanoparticles, which can be eliminated completely by thermal processing of the nanocomposite in vacuum at temperatures of up to $2800^{\circ} \mathrm{C}$ [10].

In this paper we present our measurements of electric resistance versus tem- 
perature and magnetoresistance of bulk nanocomposite samples fabricated by pressing the initial powder of carbon fishbone nanotubes. The structure of the carbon phase in the initial powder was imaged by a Philips EM 430ST transmission electron microscope of high resolution at an accelerating voltage of $200 \mathrm{kV}$. These measurements demonstrated that the major part of the initial carbon material was multilayer carbon nanotubes with lengths of several micrometers, outside diameter of 40-50 nm, and internal channel diameter of 9-20 nm. The tubes consisted of almost rectilinear sections with lengths of 100-300 nm turned with respect to one another. Figure 1 shows as an example electron micrographs of the composite nanotube material at (a) low, (b) medium, and (c) high resolution.

(See separate attached jpg files)

Fig. 1. Electron micrographs of nanotubes in the composite material at (a) low, (b) intermediate, and (c) high resolution.

The analysis of micrographs indicated that the nanotube walls were composed in most cases of 40-65 tapered graphite layers. The taper angle varied along the tubes in the range of $16-35^{\circ}$. The inside diameter was also variable. The dimensions and shapes of wider sections of the inside channel corresponded to those of catalytic nickel nanoparticles, which were detected in most cases at the ends of the nanotubes. We observed either so-called bamboo structures (with taper angles of 20 to $25^{\circ}$ ) or, more frequently, fishbone structures with larger taper angles.

Bulk samples that could be used in transport measurements were fabricated by cold pressing of nanotube powder under high ( $\sim 25 \mathrm{kbar})$ pressure. Samples were shaped as bars with dimensions of $\sim 1 \times 2 \times 3 \mathrm{~mm}$. Contacts for measuring current and voltage across samples were made from a conducting epoxy paste. Note that the samples were fairly strong and their resistivity at room temperature was relatively low: $\rho(300 K) \sim 1 \Omega \mathrm{cm}$. The resistance was measured as a function of temperature down to the liquid-helium temperature in magnetic fields of up to $75 \mathrm{kOe}$.

In all samples under investigation, the resistance changed with temperature most rapidly (about one order of magnitude) in the temperature range between liquid helium and $\sim 100 \mathrm{~K}$, and the resistance followed the law

$$
R(T)=R_{0} \exp \left[\left(T_{0} / T\right)^{1 / 3}\right]
$$

which is typical of variable-range hopping conductivity in two dimensions. Figure 2 shows as an example two curves of $\ln R$ vs. $T^{-1 / 3}$ plotted for samples Nos. 14 and 15. 


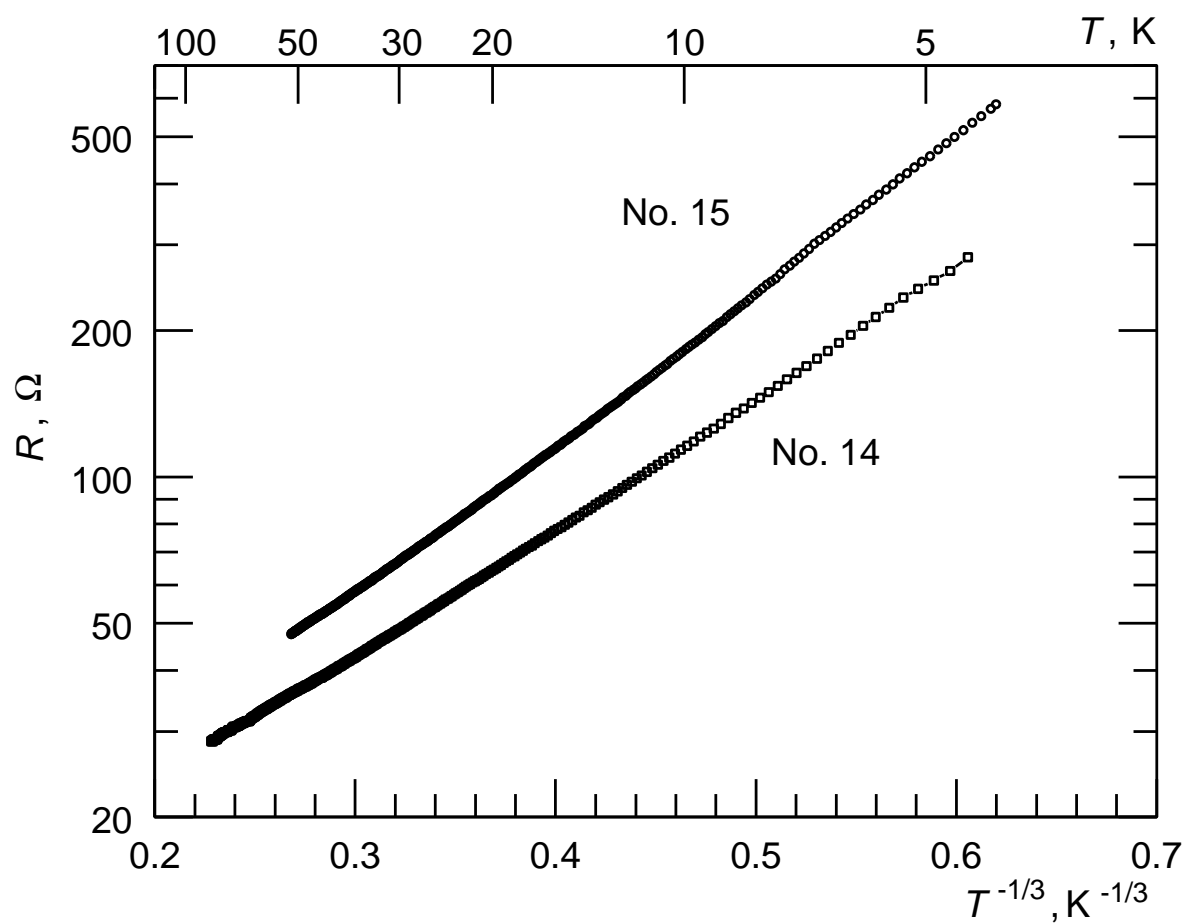

Fig. 2. Logarithmic resistance of samples Nos. 14 and 15 as a function of $T^{-1 / 3}$.

It is known [12] that in this case $T_{0}$ in Eq. (1) is given by

$$
T_{0}=\frac{13.8}{k_{B} g^{*}(\mu) a^{2}}
$$

where $g^{*}(\mu)$ is the two-dimensional density of states at the Fermi level and $a$ is the localization length.

To the best of our knowledge, this is the first observation of the dependence $\ln R \propto\left(T_{0} / T\right)^{1 / 3}$ in a system with a relatively low resistivity. Another interesting feature of our measurements is low $T_{0}$ (for example, we found $T_{0}=$ $7,3 \mathrm{~K}$ in sample No. 15 , and in all tested samples $T_{0}$ was within the interval of $6.5-7.5 \mathrm{~K}$ ), which directly indicates, in accordance with Eq. (2), that the density of states at the Fermi level is high.

In this connection, it is of interest to measure the magnetoresistance, especially as a function of temperature, since these measurements would allow us to estimate directly the localization length $a$ and then derive the two-dimensional density of states $g^{*}(\mu)$ using Eq. (2).

It is known [12] that in systems with variable-range hopping conductivity, the magnetoresistance is positive and (in moderate magnetic fields) is given by 
the expression

$$
\ln \left[\frac{\rho(H)}{\rho(0)}\right]=t\left(\frac{a}{\lambda}\right)^{4}\left(\frac{T_{0}}{T}\right)^{3 / p} \equiv A(T) H^{2}
$$

where $\lambda$ is the magnetic length, $t$ is a dimensionless factor of about 0.0025 , and $p=D+1$ (where $D$ is the system dimensionality). Since $p=3$ holds in the case under consideration, it follows from Eq. (3) that the magnetoresistance at a fixed magnetic field should be inversely proportional to the temperature.

An example of magnetoresistance measurements versus magnetic field at $T=$ $4.2 \mathrm{~K}$ for sample No. 15 is given in Fig. 3. One can see that the magnetoresistance is adequately described by a quadratic function of $H$ in the range of moderate magnetic fields, $H<30 \mathrm{kOe}$, and in higher magnetic fields it tends to a linear function.

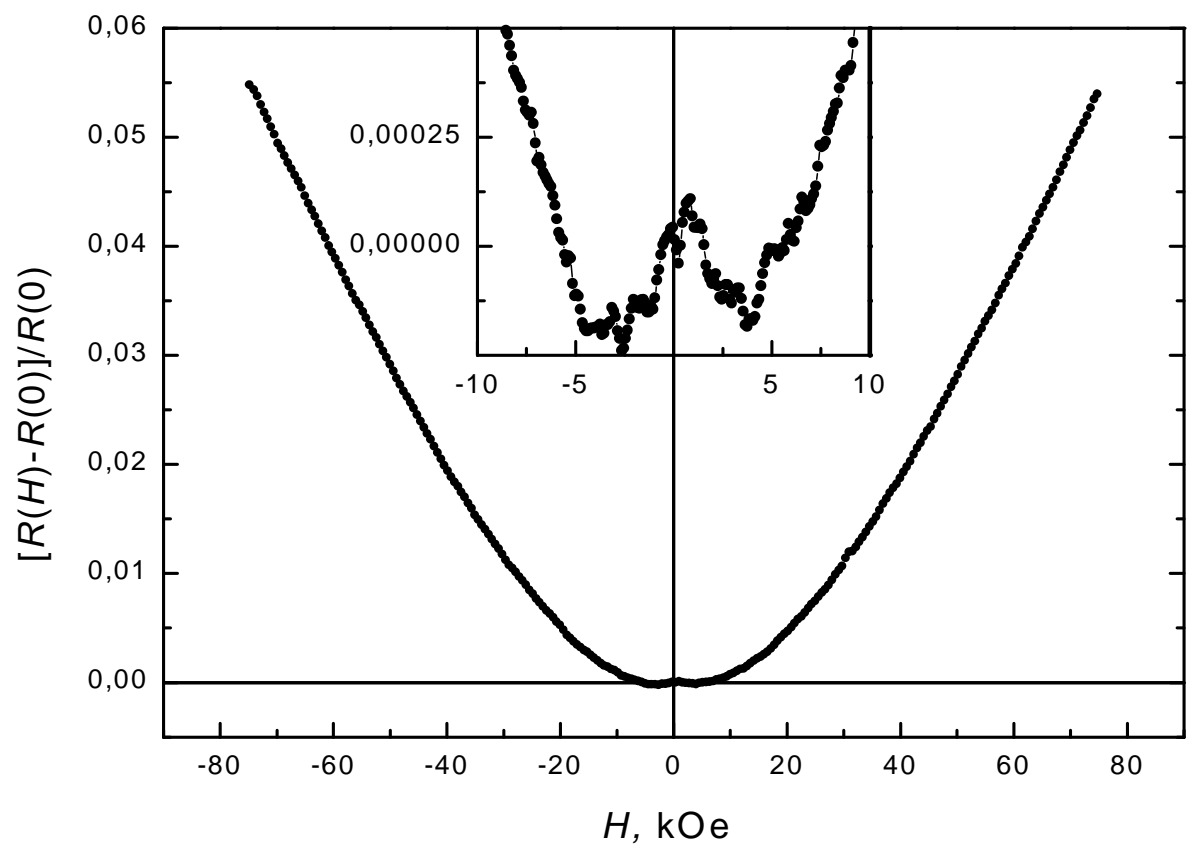

Fig. 3. Magnetoresistance of sample No. 15 versus magnetic field at $T=4.2 \mathrm{~K}$. The inset shows the section of negative magnetoresistance at low magnetic fields on an extended scale.

The behavior of magnetoresistance in low magnetic fields is especially interesting. As a rule, the magnetoresistance is negative on the section of the curve around zero and becomes positive in fields higher than $7 \mathrm{kOe}$. As a result, we have a small, broad region of negative resistivity at about 3-4 kOe. Moreover, several additional narrow local minima (see the inset to Fig. 3) are observed superposed on this broad peak. Note that the peaks in the inset to Fig. 3 are not caused by noise, although their amplitudes are very small. Experiments with repeated accumulation and averaging of the signal dedicated to testing 
the reproducibility of such measurements were performed (the results obtained by this procedure are the ones plotted in the inset to Fig. 3), and these experiments proved that the curves were reproducible, even after warming the samples to the room temperature. It seems that the negative magnetoresistance of the samples and local minima are due to the discrete structure of the conducting network formed by nanotubes. The broadest minimum in the magnetoresistance at $3-4 \mathrm{kOe}$ is tentatively related to the average cell dimension in the network, and local minima are ascribed to some additional characteristic dimensions in the random network. When the applied magnetic field reaches a value such that the magnetic flux through a network cell equals the magnetic flux quantum $h c / e$, the amplitude of the tunneling between nanotubes increases, which causes a drop in the total resistance of the system. A simple estimate yields a cell dimension of the conducting network of about $120 \mathrm{~nm}$ at $H_{\min } \sim 3.5 \mathrm{kOe}$, which seems plausible, given the structure of the nanotube material shown by the electronic microscope.

The magnetoresistance of sample No. 15 as a function of temperature under a magnetic field of $75 \mathrm{kOe}$ is plotted in Fig. 4 in terms of $\ln [R(H) / R(0)]$ and $T^{-1}$. It is clear that the magnetoresistance at low temperatures is reasonably well described by a linear function of $T^{-1}$, in accordance with Eq. (3). The localization length derived from these measurements is $a=17 \mathrm{~nm}$. Thus, the two-dimensional density of states at the Fermi level estimated using these data and Eq. (2) is $g^{*}(\mu) \sim 7.5 \times 10^{15} \mathrm{eV}^{-1} \mathrm{~cm}^{-2}$.

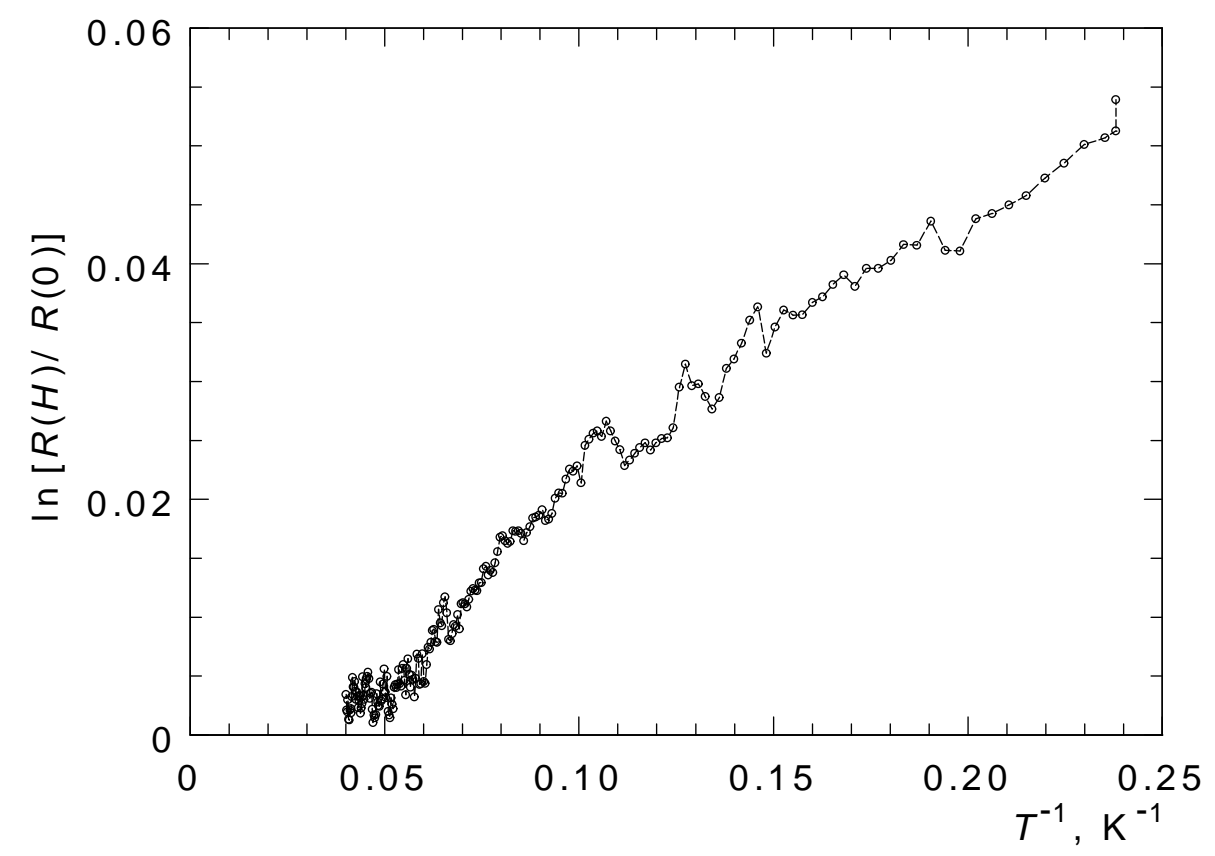

Fig. 4. Magnetoresistance of sample No. 15 versus temperature in a magnetic field of $75 \mathrm{kOe}$ plotted in coordinates $\ln [R(H) / R(0)]$ and $T^{-1}$.

Assuming that the space between the inside and outside walls of nanotubes 
acts as a two-dimensional medium, we can estimate the three-dimensional density of states $g(\mu)$ at the Fermi level. Using the relation $g^{*}(\mu)=g(\mu) d$, where $d$ is the average nanotube wall thickness (in this specific case it is about $15 \mathrm{~nm}$ ), we have $g(\mu) \sim 5 \times 10^{21} \mathrm{eV}^{-1} \mathrm{~cm}^{-3}$.

It seems also interesting to estimate the two-dimensional and three-dimensional densities, $n_{S}$ and $n_{V}$, of current carriers. This can be done using the equation

$$
n_{S}=2 g^{*}(\mu) \epsilon_{0}(T)
$$

where $\epsilon_{0}(T)$ is the energy band near the Fermi level containing current carriers contributing to the hopping conductivity [12]. In the two-dimensional case, this band width is given by the equation

$$
\epsilon_{0}(T)=\frac{\left(k_{B} T\right)^{2 / 3}}{\left[g^{*}(\mu) a^{2}\right]^{1 / 3}} .
$$

At $T=25 \mathrm{~K}$ we find from Eqs. (4) and (5) $n_{S} \sim 9 \times 10^{12} \mathrm{~cm}^{-2}, n_{V} \sim$ $6 \times 10^{19} \mathrm{~cm}^{-3}$.

Thus, we have interpreted the low-temperature transport measurements of pressed samples of randomly distributed carbon nanotubes with a nested-cones structure in terms of the two-dimensional variable-range hopping conductivity. We have assumed that the space between the inside and outside walls on nanotubes acts as a two-dimensional medium. In our previous publication [5] the low-temperature properties of carbon nanotubes were interpreted in terms of the three-dimensional model of hopping conductivity with a Coulomb gap in the density of states near the Fermi level. In both cases, the resistance is described at low temperatures by the law $\ln \rho \propto\left(T_{0} / T\right)^{1 / n}$ with small $T_{0}$, which implies that these carbon nanotube materials, with their various morphologies, are characterized by very high densities of electron states at the Fermi level of $\sim 10^{21} \mathrm{eV}^{-1} \mathrm{~cm}^{-3}$, which is a value typical of metals. This result is important for understanding the fundamental electronic properties of carbon nanotubes and related materials and may also prove quite useful from the viewpoint of practical applications.

The work was supported by the Russian Scientific Technological Program Topical Issues in Physics of Condensed Media, branch Fullerenes and Atomic Clusters (project No. 96147) and International Center for Science and Technology (project No. 079).

\section{References}


[1] M.S. Dresselhaus, G. Dresselhaus, and P.C. Eklund, Science of Fullerenes and Carbon Nanotubes, Academic Press, (1996), p.814.

[2] L. Langer, V. Bayot, E. Grivei et al., Phys. Rev. Lett. 76, 479 (1996).

[3] H. Dai, E. W. Wong, and C. M. Lieber, Science 272, 523 (1996).

[4] T. W. Ebbesen, H. J. Lezec, H. Hiura et al., Nature 382, 54 (1996).

[5] O. E. Omel'yanovskii, V. I. Tsebro, O. I. Lebedev et al., JETP Lett. 62, 503 (1995).

[6] N. A. Kiselev, O. I. Lebedev, A. N. Kiselev et al., Inst. Phys. Conf. Ser. 146, 65 (1995).

[7] Y. Saito and T. Yoshikawa, J. Crystals Growth 134, 154 (1993).

[8] M.S. Dresselhaus, G. Dresselhaus, and P.C. Eklund, Science of Fullerenes and Carbon Nanotubes, Academic Press, (1996), p.777.

[9] E. F. Kukovitskii, L. A. Chernozatonskii, S. G. L'vov, and N. N. Melnik, Chem. Phys. Lett. 266, 323 (1997).

[10] N. A. Kiselev, D. N. Zakharov, J. Sloan et al., Mol. Mat. 10(1-4) (1998).

[11] E. F. Kukovitskii, S. G. L'vov, and N.A. Sainov, Mol. Mat.10(1-4) (1998).

[12] B. I. Shklovskil and A. L. Éfros, Electronic Properties of Doped semiconductors, Springer, New York (1984) [Russ. orig. Nauka, Moscow(1979)].

Translation provided by Russian Editorial office. 
This figure "figure1a.jpg" is available in "jpg" format from: http://arxiv.org/ps/cond-mat/9812384v1 
This figure "figure1b.jpg" is available in "jpg" format from: http://arxiv.org/ps/cond-mat/9812384v1 
This figure "figure1c.jpg" is available in "jpg" format from: http://arxiv.org/ps/cond-mat/9812384v1 